\title{
A Double Hardening Model for Structured loess based on the Comprehensive Structural potential
}

\author{
LUO Ai-zhong
}

(School of architecture Engineering, Bijie University, Bijie, Guizhou 551700, China)

\begin{abstract}
Keywords: structured loess; comprehensive structural potential; double hardening model
\end{abstract}
Abstract: In order to model the macro-mechanics characters of structural loess well, and reflect the stress-strain behavior of structural loess, the structural quantitative parameter is defined based the idea of comprehensive structural potential. A double hardening model for structured loess is proposed by introducing the structural index to double hardening model for normal consolidation soil developed by professor SHEN. The rationality of structural double hardening model described here is verified by comparing the prediction results by the model proposed here with that of test for structured loess.

\section{Intruction}

Natural loess are usually structured and they differ from reconstituted-saturated loess due to their particular soil structure. For instance, they contain bonded partcles, and possibly have large void and or fissures within loess structure which are usually filled with air and water. That is, structural is a comprehensive reflection of the soil particle size, density, humidity, etc. Broadly speaking, all soils have structural. Microscopically speaking, the soil structural is interaction between soil particles, void characters, and arrangement of grains. The primary structure is constituted of solid soil particles arrangement and bond of particles each other, which is stability. When the stress that primary structure of loess is suffering is lower than structural yield stress, damage of primary soil structure is not occur, the relative position of particles ii not change or there is only small elastic deformation. Once active stress is lager than structural yield stress, micro-cracks will appears firstly on the bond of part soil particles, and then, particles sliding appears. With development of stress that soil units are suffering, micro-cracks and displacement of soil particles continue to develop. Finally, breakage and damage of aggregate particles appears, until initial structure strength was destruction completely. At the same time, new secondary structure began to form due to the mutual movement and compaction of soil particles. In the process primary structure damaging and secondary structure formation, if some impacts of the former is more than the later, soil structure would failure and softening gradually, and the complete damage would arise eventually. Recently, there have been important developments in studying the characters of structured clays, one reported effect is that natural structure conveys strength to natural soils, allowing them to exist at a higher void ratio than the equivalent remold soils at a given stress(Burland, 1990; Leroueil \& Vaughan, 1990). Sometimes loading and water content increasing causes a breakdown of the initial structure, also called destructuration, and this is particularly true in soft clays and loess (e.g. Mitchell,1970; Callisto \& Calabresi, 1998; Smith et al., 1992; Xie et al., 1999; Shao et al., 2004; Luo, 2005; Chen, 2006; Deng, 2009). Even though natural structure is apparently to model, much progress has been achieved recently in rationalizing the behavior of natural soils, such as those proposed by Gens and Nova (1993), Whittle(1993), Shen(1993,1995), Wheeler (1997), Rouainia and Muir Wood (2000), and Kavvadas and Amorosi (2000), Liu(2000), He and Shen(2002), Luo and Bai(2004), Den(2009), 
Jin and Zhao(2010), Suebsuk(2010). In this paper a new constitutive model for structured loess is proposed. The main objective of this new formulation is to provide a constitutive model suitable for solution of boundary value problems encountered in geotechnical engineering practice of loess region. It is intended therefore, that the new model should be contained impact of water content and stress, and the each other of parameters has a clear physical meaning and be conveniently identified. The model has also been used to predict the behavior of variety of structured loess in both compression and shearing tests, allowing for a reasonably comprehensive evaluation. It is demonstrated that the proposed model is suitable for describing the behavior of a variety of natural structured loess.

\section{The comprehensive structural potential and evolution of the strength parameters}

Xie(1999) proposed a quantitative parameter to evaluate the structural of structured loess, which is called comprehensive structural potential, based on summarizing the history of structural research for structured soils. The biggest advantage of comprehensive structural potential is that it can describe the true response due to the structural variation of loess in external conditions changing. Later, many structural parameters are proposed based comprehensive structural potential(Shao,2004; Luo,2005; Chen, 2006; Feng, 2008; Deng,2009). Deng(2009) proposed a new stress ratio structure parameter, which can reflect the coupling action of shear stress and mean spherical stress. The stress ratio structure parameter $m_{\eta}$ was defined by

$$
m_{\eta}=\frac{(q / p)_{i} /(q / p)_{r}}{(q / p)_{s} /(q / p)_{i}}=\frac{\eta_{i}^{2}}{\eta_{r} \eta_{s}}
$$

Where, $m_{\eta}$ is the stress ratio structure parameter. $(q / p)_{i},(q / p)_{r}$ and $(q / p)_{s}$ are stress ratios of intact loess, remold loess and saturated loess in the shear process. $\eta$ is the stress ratio. The stress and strain quantities used in the present formulation are defined as follows. $\sigma_{i j}$ and $\varepsilon_{i j}$ are the Cartesian components of effective and of strain respectively. The simplified forms for stress and strain conditions in conventional triaxial tests are also listed, where $\sigma_{1}\left(\right.$ or $\left.\varepsilon_{1}\right)$ and $\sigma_{3}$ (or $\left.\varepsilon_{3}\right)$ are the axial effective stress(strain), and the radial effective stress(strain) respectively. The mean effective stress $p$, deviatoric stress $q$ and stress ratio $\eta$ are given by

$$
\begin{aligned}
& p=\frac{1}{3}\left(\sigma_{11}+\sigma_{22}+\sigma_{33}\right) \\
= & \frac{1}{3}\left(\sigma_{1}+2 \sigma_{3}\right) \text { for conventional triaxial tests, } \\
q= & \frac{1}{\sqrt{2}}\left[\left(\sigma_{11}-\sigma_{22}\right)^{2}+\left(\sigma_{22}-\sigma_{33}\right)^{2}+\right. \\
& \left.\left(\sigma_{33}-\sigma_{11}\right)^{2}+6\left(\sigma_{12}{ }^{2}+\sigma_{23}{ }^{2}+\sigma_{31}{ }^{2}\right)\right]^{1 / 2} \\
= & \left(\sigma_{1}-\sigma_{3}\right) \text { for conventional triaxial tests, }
\end{aligned}
$$




$$
\eta=\frac{q}{p}
$$

The corresponding volumetric strain increment, $d \varepsilon_{v}$, and deviatoric strain increment, $d \varepsilon_{s}$, are given by

$d \varepsilon_{v}=d \varepsilon_{11}+d \varepsilon_{22}+d \varepsilon_{33}$

$=d \varepsilon_{1}+2 d \varepsilon_{3}$ for conventional triaxial tests,(5)

and

$$
\begin{aligned}
& d \varepsilon_{s}=\frac{\sqrt{2}}{3}\left[\left(d \varepsilon_{11}-d \varepsilon_{22}\right)^{2}+\left(d \varepsilon_{22}-d \varepsilon_{33}\right)^{2}+\right. \\
& \left.\left(d \varepsilon_{33}-d \varepsilon_{11}\right)^{2}+6\left(d \varepsilon_{12}{ }^{2}+d \varepsilon_{23}{ }^{2}+d \varepsilon_{31}{ }^{2}\right)\right]^{1 / 2}
\end{aligned}
$$$$
=\frac{2}{3}\left(d \varepsilon_{1}-d \varepsilon_{3}\right) \text { for conventional }
$$

triaxial tests,

Based the test data which are derived from literature(Shao et al, 2004), the evolution of stress ratio structural parameter is obtained, as showing:

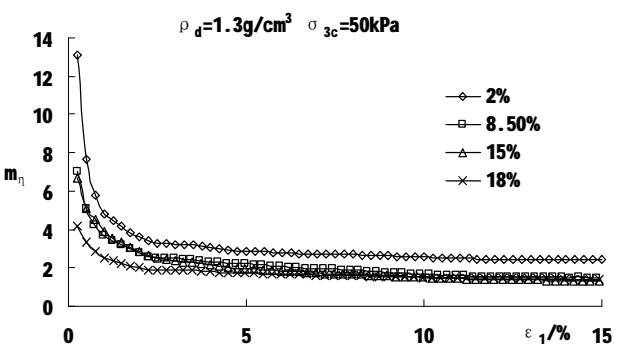

(a) $50 \mathrm{kPa}$

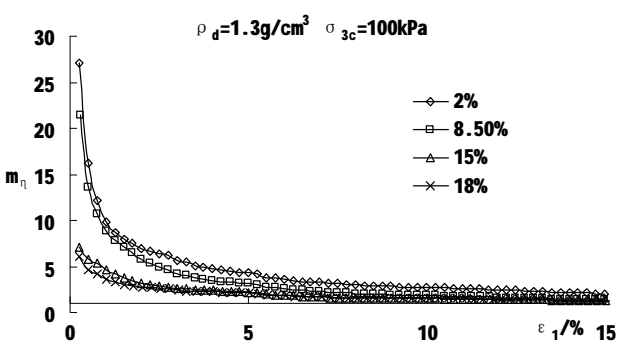

(b) $100 \mathrm{kPa}$

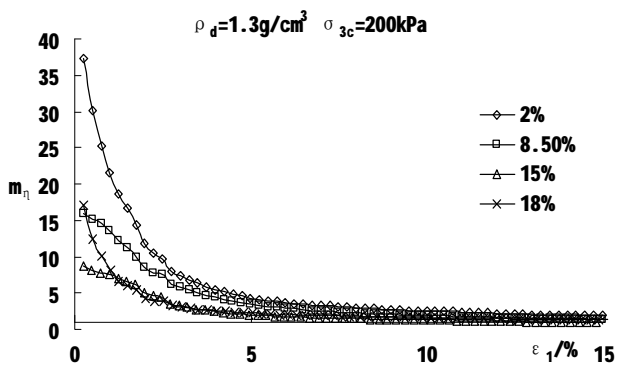

(c) $200 \mathrm{kPa}$

Fig.1 The change law between $m_{\eta}$ and $w \%$

The agreement between data, which is particularly good for structured loess, shows that when all effects of destructuration are assumed to be included in the parameter. This can be formulated as $m_{\eta}=f\left(w, \sigma_{3 c}, \varepsilon_{s}\right)=A \varepsilon_{s}^{B}+1$

where A and B are the material constants, which are related with the initial water content and initial 
consolidation pressures in shear process. A and B are calculated by

$$
\begin{gathered}
A=a_{1} e^{b_{1} \sigma_{c}} \\
B=a_{2} e^{b_{2} \sigma_{c}}
\end{gathered}
$$

and

$$
\begin{aligned}
& a_{1}=-0.6622 w+3.3999 \\
& b_{1}=-0.0002 w+0.0089 \\
& a_{2}=-0.0075 w-0.2084 \\
& b_{2}=-0.0003 w+0.0074
\end{aligned}
$$

Where, $\sigma_{c}$ and $w$ are the initial consolidation pressures and water content in the shear process. The destructuration law proposed uses basic formation given in equation (7). It is combined with the comprehensive impact of water content, consolidation pressures and shear deformation. With water content increasing and shear deformation developing, structural of loess is weaken gradually, and the stress ratio structural parameter is one finally. Based on comprehensive structural potential idea, structural of loess is dissipated fully and soil structure tends to destroy when stress ratio structural parameter is one. Fig.2 shows the relationship of stress ratio structural parameter and cohesions, Fig.3 shows the relationship of stress ratio structural parameter and friction. By analyzing the results derived from Fig.2 and Fig.3, it is found that there is exponential between the relationship of stress ratio structural parameter and cohesions, and there is little change between the relationship of stress ratio structural parameter and friction. The relationship of stress ratio structural parameter and cohesions is showed by

$$
c=E\left(1-F^{m_{\eta}}\right)
$$

where, $\quad E=288.042, \quad F=0.8874$.

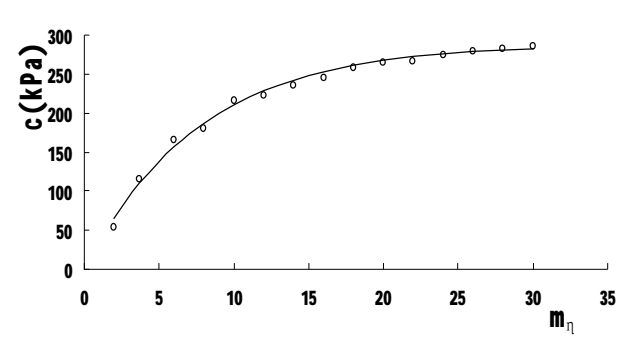

Fig.2 The relationship of between cohesion and structural index

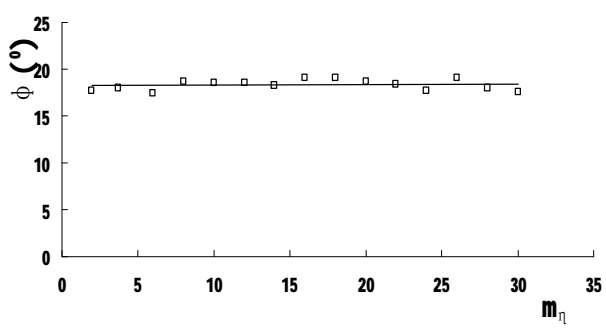

Fig.3 The relationship of between friction angle and structural index 


\section{The constitutive model considered structural of loess}

\section{Knowledge of loess structural}

Structural of soil, which is equal with density and moisture, is one of basic properties of natural sediment soil, constitutive model of soil is any mathematical description of deformation and strength of soil based on the experimental study, which is able to consider the main property of soil mechanical properties. As soil is a kind of discontinuous material, there is not only shear plastic deformation but also volume plastic deformation in the loading process. Natural loess are usually structured and they differ from reconstituted-saturated loess due to their particular soil structure. The main objective of this new formulation is to provide a constitutive model suitable for the solution of boundary value problems encountered in geotechnical engineering practice. It is intended therefore, that the new model should be relatively simple and should have few parameters, each of which has a clear physical meaning and can be conveniently identified. The model should also be relatively easy to understand and apply. Based the understanding above, the quasi-preconsoidation pressures which were related with structural of loess should be determined by compression tests, and the size of yield surface of structural loess was derived from $e \sim \ln p$ curves.

By research the characteristics between strength and structural of structural loess, the variation of critical state line in structural changing process was obtained. According to the characteristics that stress-strain curves were hyperbola by normalizing with structural parameters, it is showed that loess is still subjected to critical state soil mechanics under the same structural parameter. So the compression fearers and critical state line under same structural parameter can be induced to

reconstituted model.

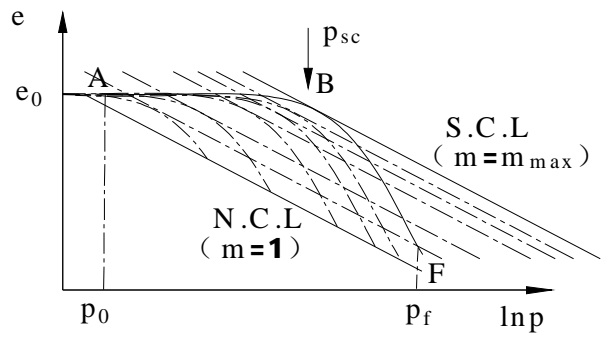

Fig.4 Curves of structural loess under different moisture

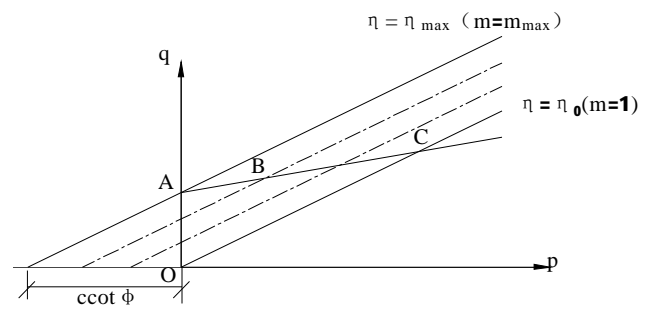

Fig.5 Shear yield surface of structural loess under different moisture

\section{A double hardening model for structural loess}

Shen provided a double hardening model for clay, which is a closed yield surface model and includes two hardening parameters: plastic volume strain and deviatoric strain. The yield surface of double hardening model not only expands and contacts with accumulating of hardening parameter, but also deformation appears with ratio difference of two parameters. So the double hardening model can describe the characteristics of normally consolidated clay and over-consolidated clay. It is intended therefore, that new model should be contained impact of structural based double 
hardening model.

The yield function is described by

$$
F=\frac{\sigma_{m}}{1-\left(\eta / \eta_{0}\right)^{n}}-H=0
$$

Where

$$
\begin{aligned}
& \sigma_{m}=\frac{1}{3}\left(\sigma_{1}+\sigma_{2}+\sigma_{3}\right)+c\left(m_{\eta}\right) \cot \varphi \\
& \eta=\frac{1}{\sqrt{2}}\left[\left(\frac{\sigma_{1}-\sigma_{3}}{\sigma_{1}+\sigma_{3}+2 c\left(m_{\eta}\right) \cot \varphi}\right)^{2}+\right. \\
& \left(\frac{\sigma_{1}-\sigma_{2}}{\sigma_{1}+\sigma_{2}+2 c\left(m_{\eta}\right) \cot \varphi}\right)^{2}+ \\
& \left.\left(\frac{\sigma_{2}-\sigma_{3}}{\sigma_{2}+\sigma_{3}+2 c\left(m_{\eta}\right) \cot \varphi}\right)^{2}\right]^{1 / 2}
\end{aligned}
$$

The hardening law is given by

$$
\begin{gathered}
H=p_{0} \exp \left(\frac{\varepsilon_{v}^{p}}{c_{c}-c_{s}}\right) \\
\eta_{0}=\eta_{1}\left[1-\alpha \exp \left(-\frac{\varepsilon_{s}^{p}}{c_{a}}\right)\right]
\end{gathered}
$$

Where, $p_{0}$ is reference stress when $\varepsilon_{v}^{p}=0 ; c_{c}=\lambda /\left(1+e_{0}\right), \quad c_{s}=\kappa /\left(1+e_{0}\right), \lambda, \kappa$ is the slope of compression curves and swell curves on $e \sim \ln p, e_{0}$ is the initial void ratio ; $\eta_{1}=n \sqrt{1+n} \sin \varphi_{r}$, $\varphi_{r}$ is friction of remold and saturated loess; $n=1.2 ; c_{a}, \alpha$ is the material parameter。

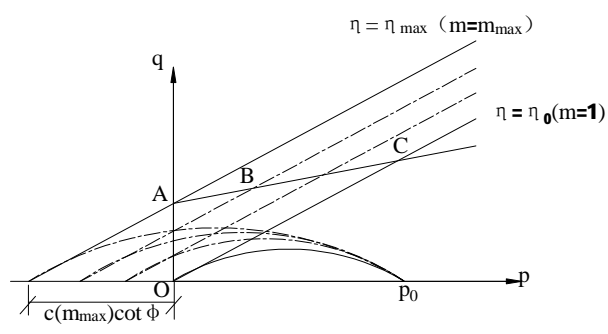

Fig.6 Double hardening yield surfaces considering structural

\section{Stress-strain relationship of structural double hardening model}

According to the consistency requirement, equation (6) is changed as:

$$
\begin{aligned}
& \frac{\partial F}{\partial \sigma} d \sigma+\frac{\partial F}{\partial \eta} \frac{\partial \eta}{\partial c} \frac{\partial c}{\partial m} \frac{\partial m}{\partial \varepsilon_{s}^{p}} d \varepsilon_{s}^{p}+ \\
& \frac{\partial F}{\partial \eta_{0}} \frac{\partial \eta_{0}}{\partial \varepsilon_{s}^{p}} d \varepsilon_{s}^{p}+\frac{\partial F}{\partial H} \frac{\partial H}{\partial \varepsilon_{v}^{p}} d \varepsilon_{v}^{p}=0
\end{aligned}
$$

And according to associative flow rule, there is

$$
d \varepsilon^{p}=\Lambda \frac{\partial F}{\partial \sigma}
$$

Based equation (11) and equation (12), there is

$$
\Lambda=-\frac{\left(\frac{\partial F}{\partial \sigma}\right)^{T} d \sigma}{A 1+A 2}
$$

where 


$$
\begin{aligned}
& A 1=\frac{3}{2}\left[\frac{\partial F}{\partial \eta} \frac{\partial \eta}{\partial c} \frac{\partial c}{\partial m_{\eta}} \frac{\partial m_{\eta}}{\partial \varepsilon_{s}^{p}}+\frac{\partial F}{\partial \eta_{0}} \frac{\partial \eta_{0}}{\partial \varepsilon_{s}^{p}}\right] \frac{\partial F}{\partial \sigma_{s}} \\
& A 2=\frac{\partial F}{\partial H} \frac{\partial H}{\partial \varepsilon_{v}^{p}} \frac{\partial F}{\partial \sigma_{m}}
\end{aligned}
$$

and then

$$
d \varepsilon^{p}=\frac{1}{A}\left\{\frac{\partial F}{\partial \sigma}\right\}\left\{\frac{\partial F}{\partial \sigma}\right\}^{T} d \sigma
$$

Where, $A$ is hardening modulus, and $A$ is described by

$$
A=A 1+A 2
$$

Elastic strain can be calculated by bulk modulus, which is

$$
B_{m}=\frac{p_{m}}{c_{s}}
$$

where, $p_{m}$ is maxinum consolidated pressure, poisson ratio is 0.25 .

\section{Simulations}

The practicality of double hardening model for many stress paths has been validated by SHEN in literature [18]. This model described herein considers the structural of loess based double hardening model. The first set of experimental data that has been simulated using the formulation developed above relates to the structural of loess. The parameters related to structural of loess would been calculated by equation (8) and equation (9). There are two tests have been simulated where the water content are $2 \%$ and $15 \%$ under consolidation pressure $100 \mathrm{kPa}$. The model parameters are shown by Table 1 .

Table 1 Calculation Parameters

\begin{tabular}{cccccccc}
\hline$c_{c}$ & $c_{s}$ & $c_{a}$ & $\eta_{1}$ & $n$ & $v$ & $E$ & $F$ \\
\hline 0.04 & 0.00 & 0.2 & 1.1 & 1. & 0.3 & 288.0 & 0.88
\end{tabular}

$\begin{array}{llllllll}4 & 5 & 5 & 6 & 2 & 0 & 4 & 7\end{array}$

Figures 7 and 8 shown the simulating results and experiment data. It shows that the model provided in this paper predicts the characteristics of loess, and the simulating results are basically consistent with the tests data. In general, the model described herein considers structural of loess, and makes the water contents are considered into the soil constitutive relations. Furtherly, the macro stress-strain characteristics are given a reasonable description.

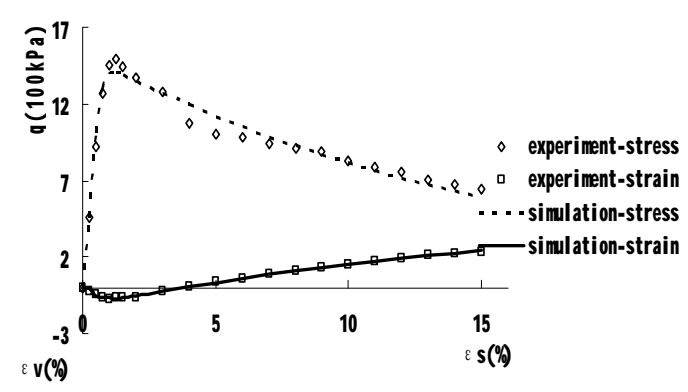

Fig.7 Comparing of stress-strain relationship when $\sigma_{3 c}=100 \mathrm{kPa} 、 w=2 \%$ 


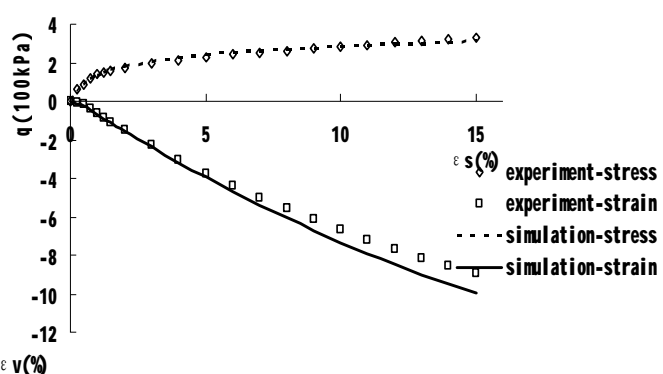

Fig.8 Comparing of stress-strain relationship when $\sigma_{3 c}=100 \mathrm{kPa} 、 w=15 \%$

\section{Conclusions}

(1) Based the idea of comprehensive structural potential, the comprehensive potential quantification parameter is provided, which is power function with generalized shear strain.

(2) The relationship between structural parameters and strength parameters is considered, and at the same whose mathematical description is given.

(3) The structural double hardening model is provided by considering the structural effect based double hardening model for clay.

(3) The reasonability of structural double hardening model is verified by analysing the predicted results and experimental data of structural loess, and this model can predict the macro stress-strain behavior of structural loess.

\section{Peferences:}

[1] GONG Xiao nan, XIONG Chuan xiang, XIANG Ke xiang et al. The formation of clay structure and its influence on mechanical characteristics of clay[J], Journal of Hydraulic Engineering, 2000,10:43-47.

[2] HU Rui Lin, Research on Quantitative Microstructure Models of Natural clayed soils and its Engineering Geology characteristics[M], Bei Jing: BeiJing Geological Publishing House, 1995.

[3] Xie Dingyi, Yao Yangping, Dang Faning, Advanced Soil Mechanics, BeiJing: Higher Education Press,2008.

[4] Baudet B, Stallebrass S. A constitutive model for structured clays. Geotechnique 2004;54(4):269-78.

[5] Burland, J.B. 1990. 30th Rankine Lecture: On the compressibility and shear strength of natural clays. Géotechnique 40(3): 327-378.

[6] Kavvadas, M. \& Amorosi, A. 2000. A constitutive model for structured soils. Géotechnique 50(3): 263-274.

[7] Leroueil, S., Tavenas, F., Brucy, F., La Rochelle, P. \& Roy, M. 1979. Behaviour of destructured natural clays. Journal of Geotechnical Engineerig Div. ASCE 105(6): 759-778.

[8] Rouainia, M. \& Muir Wood, D. 2000. A kinematic hardening constitutive model for natural clays with loss of structure. Géotechnique, Vol. 50(2): 153-164.

[9] Liu, M.D., and Carter, J.P. 1999. Virgin compression of structured soils. Géotechnique, 49(1): 43-57.

[10] Liu, M.D., and Carter, J.P. 2000. Modelling the destructuring of soils during virgin compression. Géotechnique, 50(4): 479-483.

[11] Suebsuk J, Horpibulsuk S, Liu MD. Modified structured Cam Clay: a generalized critical state 
model for destructured, naturally structured and artificially structured clays. Comput Geotech 2010;37(7-8):956-68.

[12] Shen Zhujiang.An Eiasto-piastic Damage Model for Cemented Clays[J] Chinese J. Geot. Eng., 1993,V15(3): 21-28.

[13] LIU En-long, SHEN Zhu-jiang, Modeling compression of structured soils[J], Rock and Soil Mechanics, 2006,27(4): 615-620

[14] HE Kai-sheng, SHEN Zhu-jiang, Elasto-viscoplastic damage model for structural clays[J], Hydro-science and engineering, 2002,4

[15] LUO Xiao-hui, BAI Shi-wei, Model analysis on statistical damage of strength of cemented soil, Chinese Journal of Geotechnical Engineering, 2004, 26(5): 712-714.

[16] JIN Xu, ZHAO Cheng-gang, LIU Yan et al, An elasto-plastic damage constitutive model of unsaturated soils[J], Journal of BeiJing Jiao Tong University, 2010,34(1):78-82

[17] HU Zai-qiang, SHEN Zhu-jiang, XIE Ding-yi, Constitutive model of structural loess[J], Chinese Journal of Rock mechanics and Engineering, 2005,24(4):17-21.

[18] SHEN Zhu-jiang, A Double Hardening Model for clays, Rock and Soil Mechanics, 1995,16(1): $1-8$

[19] XIE Ding-yi, QI Ji-li. Soil structure characteristics and new approach in research on its quantitative parameter [J]. Chinese Journal of Geotechnical Engineering, 1999, 20(2): 651-656.

[20] SHAO Sheng-jun, ZHOU Fei-fei,etal.. Structural properties of loess and its quantitative parameter [J]. Chinese Journal of Geotechnical Engineering, 2004, 26(4): 531-536.

[21] CHEN Cun-li ,Hu zai-qiang,etal.Research on relationship between structure and deformation property of intact loess[J]. Rock and Soil Mechanics, 2006,27(11):1891-1896.

[22] Feng zhi-yan. Research on Unsaturated Loess structural quantitative parameter and structural Constitutive [D], Xi'an: Institute of Geotechnical Engineering in Xi'an University of Technology, 2008

[23] Luo ya-sheng,Xie ding-yi,etal.Structural Constitutive Relation of Soils Under Complex Stress Conditions[J]. Journal of Sichuan University (Engineering Science Edition,2005,37(5):14-17.

[24] Deng guo-hua. Research on strength and deformation constitutive relations of structured soils under true tri-axial condition [D], Xi'an: Institute of Geotechnical Engineering in Xi' an University of Technology,2009. 